\title{
Effective Reinforcement of Carbon-Carbon Composites Using Morped Graphene
}

\author{
H. A. Calderon ${ }^{1}$, D. A. Barber ${ }^{2}$, Fernando Alvarez Ramirez ${ }^{3}$, R. Ordoñez Olivares ${ }^{4}$, V. Hadjiev ${ }^{5}$ and F. \\ C. Robles Hernandez ${ }^{2}$
}

${ }^{1}$ Depto. de Física, ESFM-IPN, Ed. 9 UPALM, Mexico D.F., Mexico.

2. Mechanical Engineering Technology Department, University of Houston, TX.

${ }^{3 .}$ Instituto Mexicano del Petróleo, Eje Central Lázaro Cárdenas Norte 152, México D.F., Mexico.

4. University of Pittsburg, Mech. \& Mats. Sci. \& Eng. Dept., Pittsburgh, PA.

5. Texas Center for Superconductivity, University of Houston, Houston, TX.

This work describes a novel method to synthesize two newly identified phases of carbon known as morphed graphene. The morphed graphene have the Rh6 and Rh6-II structures that are synthesized purely by milling of amorphous carbon sources. Depending on the synthesis route large amounts of double or triple layer graphene can be synthesized as well. The morphed graphene is a rather more complex structure than graphene alone. In morphed graphene the graphitic layers have crosslinking capabilities along the "c" direction. The Rh6 is purely sp2 and the Rh6-II is pure sp3. The synthesis of the morphed graphene is purely mechanical and therefore it is green and at room temperature. The main novelty of the work is not only the synthesis of the Rh6 and Rh6-II pases, but also their understanding along with their phase transformations by means of Low Dose Transmission Electron Microscopy. The morphed graphenes were previously proposed numerically, but as of today, we are the only group that has successfully synthesized them [1-5]. Once the morphed graphene are synthesized they are sintered using spark plasma sintering methods to produce the composites. The sintering further increase the presence of those new structures forming networks that act as effective reinforcements resulting in quite outstanding mechanical properties. The sintered composites are merely elastic and under quite severe conditions they show outstanding plasticity and recovery. Depending on the synthesis route we can produce up to $20 \% \mathrm{sp} 3$ at room temperature that be increased to more than $40 \%$ in-situ during sintering. The presence of $\mathrm{sp} 2$ and $\mathrm{sp} 3$ is controlled mainly during the milling process.

The characterization technique that is the most important herein this work is the Low Dose Transmission Electron Microscopy. The low dose was particularly of interest because this method does not generate any damage to the materials under observation. The atomic resolution transmission electron microscopy imaging was carried in the TEAM 0.5 microscope with aberration-correction at $80 \mathrm{kV}$. The experimental images were taken with a spherical aberration coefficient of $-0.015 \mathrm{~mm}$ with a focus spread of $\sim 10 \AA$ and in conditions of low dose. The dose rate has been reduced to around $20 \mathrm{e}^{-} / \AA^{2} \mathrm{~s}$ for images in focal series. A focal series is used to reconstruct the exit wave (EWR) and determine both phase and amplitude images using MacTempas ${ }^{\circledR}$. A phase image contains the information regarding both nature and spacings of the atomic columns in the sample volume. A focal series consists of 40 images taken with an exposure time of $1 \mathrm{~s}$ and at different focus settings, between each image there is a waiting time of approximately $1 \mathrm{~s}$. Thus the total dose in a focal series reaches approximately $800 \mathrm{e}-/ \AA 2$. Such a dose is sufficiently low to prevent any damage as a result of beam sample interaction in this investigation. A normal alignment of the TEAM 0.5 has been done by keeping aberrations such as $\mathrm{C} 1, \mathrm{~A} 1, \mathrm{~A} 2, \mathrm{~B} 2, \mathrm{~A} 3$, S3, A4, D4, B4, C5 and A5 within the confidence limits of measurement and keeping C3 at the specified value. The conditions have been earlier tested in different materials to image the genuine atomic arrangement of the sample by preventing any damage and keeping the required resolution of the investigated material. Figure 1 shows the low dose images and Figure 2 shows the mechanical testing result of the composites. The acknowledgements of this work are in [6]. 
References:

[1] H.A. Calderon et al, Adv Struct Chem Imaging 2 (2017), p. 10.

[2] I. Estrada-Guel et al, MRS Proceedings 1765 (2015), p. 29.

[3] H.A. Calderon et al, Carbon 102 (2016), p. 288.

[4] J.-T. Wang et al, Physical Review Letters 116 (2016), p. 195501.

[5] J.-T. Wang et al, Scientific Reports 4 (2014), p. 4339.

[6] C.f.a.s.s.a.t.M.F.-L.W.a.t.M.F.w.s.b.t.O.o.S. HAC acknowledges support from IPN (SIP, Office of Basic Energy Sciences, of the U.S. Department of Energy under Contract No. DE-AC02-05CH1123.

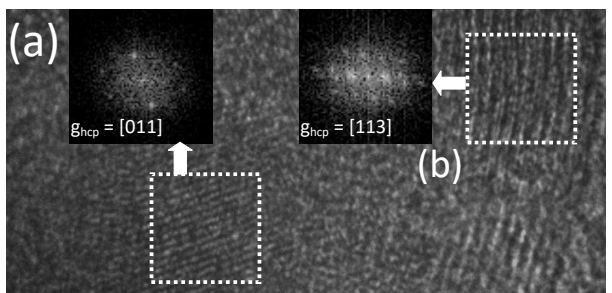

(c)

\section{$5 \mathrm{~nm}$}

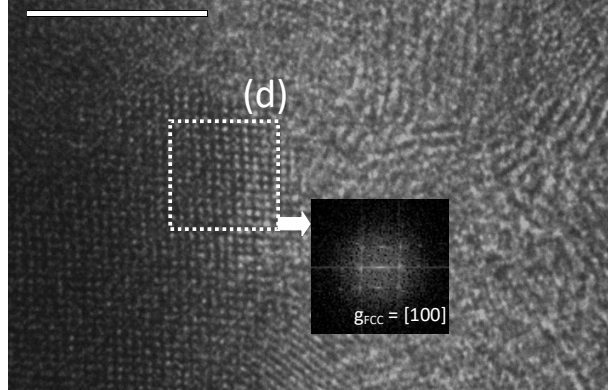

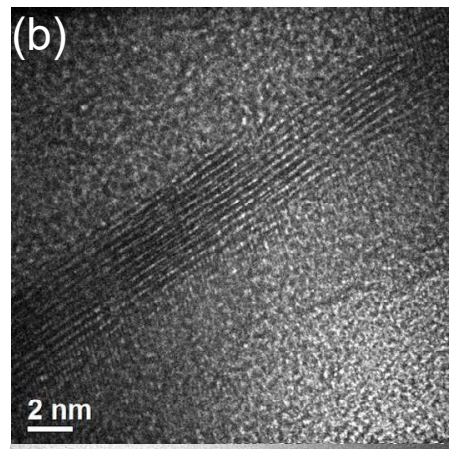

(c)

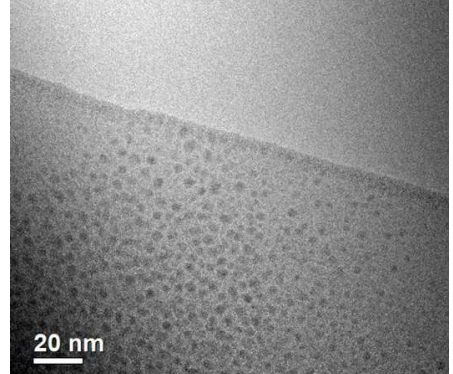

Figure 1. AC-TEM under low dose conditions of the (a) milled product and (b-c) sintered product at various times of milling. Note that the morphed graphene in (b) is the Rh6 and the one in (c) is the Rh6-II.

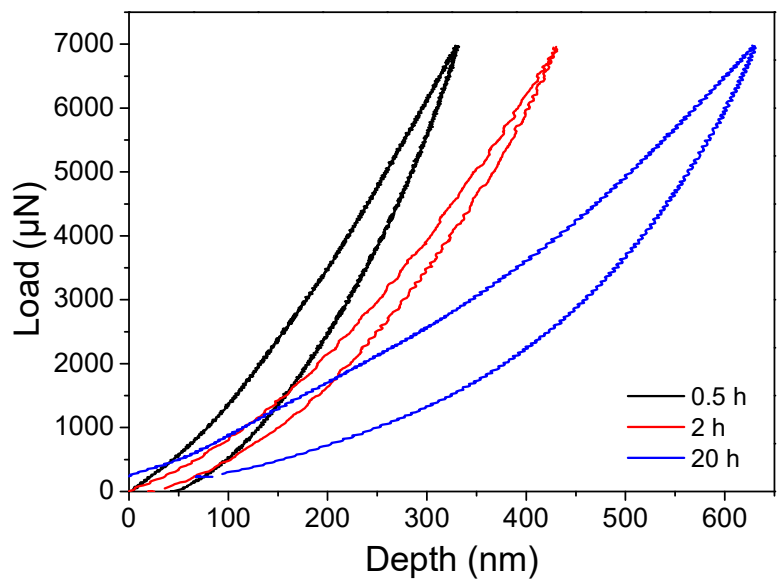

Figure 2. Mechanical characterization of the morphed graphene composites under nanohardness testing. 